CERN-TH/99-85

hep-th/9903232

\title{
On the Anomalous and Global Interactions of Kodaira 7-Planes内
}

\author{
W. Lerche and S. Stieberger \\ Theoretical Physics Division, CERN \\ 1211 Geneva 23, Switzerland
}

\begin{abstract}
:
We review interactions between certain 7-planes which are composed out of mutually non-local $(p, q)$ 7-branes and which correspond to specific Kodaira singularities. We discuss in particular how to compute certain moduli-dependent terms in the effective action. These do not only probe the local Chern-Simons terms on the world-volumina, but also certain global aspects of plane interactions that can be attributed to "torsion" (or

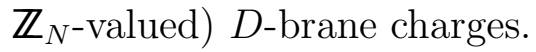

\section{Introduction}

When studying interactions between $D$-branes, one usually considers idealized situations where one focuses one a single pair of (possibly stacks of) branes. However, for making quantitative tests of string dualities, the global structure of $D$-branes interactions become important as well - that is, the influence of all the other branes that are usually considered as far away. The purpose of this contribution is to elucidate some aspects of such "global interactions", reviewing part of ref. [1] but also slightly expanding upon some of the points.

The theory we are concretely interested in here is $F$-theory compactification on $K 3$, which amounts to type IIB compactification on $\mathbb{P}^{1}$ with 247 -branes [2]. This theory is dual to the heterotic string on $T^{2}$, where certain anomaly-related pieces of the effective action can be computed exactly at one-loop order. This fact provides an ideal framework for studying non-trivial brane interactions; in the past, it has been very successfully applied to study brane interactions in type I and matrix strings (see eg., [3, 4, 5]).

Obviously, one cannot expect to compute any given piece of the effective lagrangian exactly; it is just very special couplings, namely typically those which are anomaly-related and/or to which only BPS-states contribute, that are amenable to an exact treatment. In the present situation with 16 supercharges in eight dimensions, the canonical BPSsaturated amplitudes [6, 7] involve four external gauge bosons (and/or gravitons). Supersymmetry relates parity even $\left(i \xi F^{n}\right)$ and parity odd $\left(\frac{\theta}{2 \pi} F \wedge F \wedge . . F\right)$ sectors, and therefore one can conveniently combine the theta-angle and the coupling constant $\xi$ into

\footnotetext{
${ }^{1}$ Contribution to the proceedings of the 32nd International Symposium on the Theory of Elementary Particles, Buckow 1998, to appear in "Fortschritte der Physik".
} 
one complex coupling, $\tau_{\text {eff }}$. In particular, when compactifying the heterotic string on $T^{2}$, the effective couplings $\tau_{\text {eff }}(T, U) \equiv i \xi(T, U)+\frac{1}{2 \pi} \theta(T, U)$ become highly non-trivial functions depending on the usual torus moduli $T$ and $U$. As mentioned before, in the heterotic string picture the couplings are exact at one-loop order, and are in fact directly related [6] to the heterotic elliptic genus $\widehat{\mathcal{A}}(F, R, q)$ [8]. Schematically:

$$
\left.\operatorname{Re}\left[\tau_{\text {eff }}(T, U)\right] F \wedge \ldots F \sim \int \frac{d^{2} \tau}{\tau_{2}} Z_{(2,2)}(T, U, q, \bar{q}) \widehat{\mathcal{A}}(F, R, q)\right|_{8-\text { form }} \quad
$$

where $Z_{(2,2)}$ is the partition function of the two-torus $T^{2}$ (we will switch off the Wilson lines, or set them to certain constant values). The evaluation of such integrals has been discussed at length in [9, औ, 1, 10, and results in certain Borcherds-type of modular functions. The resulting expressions have been interpreted in terms of type I string language [3, 4, 5], in which one has been able to identify both perturbative and nonperturbative ( $D$-instanton) contributions. On the other hand, an interpretation in terms of $F$-theory geometry was given in refs. [1, 10, 11], and this is what we will -partiallyreview below.

\section{Chern-Simons Couplings on 7-Planes}

The issue is to compute the functions $\tau_{\text {eff }}(T, U)$ (1) via 7-brane interactions. While in general very complicated, these interactions in the present context reasonably tractable because of their special anomaly related, parity-odd structure. They arise from the ChernSimons terms on the world-volumina of the 7-branes, via the exchange of $R R$ antisymmetric tensor fields $C^{(p)}$. For a single $D$-brane, the relevant tree level couplings look [12] (for trivial normal bundle):

$$
\mathcal{L}_{D 7}^{(\mathrm{CS})}=\left.C \wedge e^{-2 i F} \wedge \sqrt{\widehat{\mathcal{A}}(R)}\right|_{8-\text { form }}
$$

where $C \equiv \oplus_{k=0}^{4} C^{(2 k)}$ is the formal sum over all $R R$ forms, and $\widehat{\mathcal{A}}(R)$ is the Dirac genus.

Note that due to the generic mutual non-locality of the $24(p, q) 7$-branes, it is a priori not clear how to add up these terms, and much less, how to determine what effective interactions they induce. However, what we can do is simply to restrict to sub-moduli spaces where the 24 branes combine into certain objects ("7-planes") that are all mutually local, ie., have commuting monodromies. Particularly simple are the sub-cases where the monodromies not only commute, but are also of finite order. These correspond to theories where the 7-brane charge is cancelled locally, such that the type IIB string coupling $\tau_{S}$, is constant over the $\mathbb{P}^{1}$ base (the " $z$-plane"). There are three such possibilities for splitting up the 24 7-branes [13, 14]:

ii) $\tau_{S}=i$ : into eight $\mathcal{H}_{1}$-planes (five remaining moduli)

ii) $\tau_{S}=\rho \equiv e^{2 \pi i / 3}$ : into twelve $\mathcal{H}_{0}$-planes (nine remaining moduli)

iii) $\tau_{S}=$ arbitrary constant: into four $\mathcal{D}_{4}$-planes (one remaining modulus; this branch intersects branches ii) and iii)).

Here $\mathcal{H}_{n}$ denotes 7-planes associated with the Kodaira elliptic singularity types [15] of the same name; they carry gauge symmetries of type $A_{n}\left(A_{0} \sim U(1)\right)$, respectively. By further specialization, one can have some or all of the $\mathcal{H}_{n}$ branes combine into planes with larger gauge symmetries; the various possibilities are listed in the following table. 


\begin{tabular}{|c|ccccccc|}
\hline Kodaira type $\rightarrow$ & $\mathcal{H}_{0}$ & $\mathcal{H}_{1}$ & $\mathcal{H}_{2}$ & $\mathcal{D}_{4}$ & $\mathcal{E}_{6}$ & $\mathcal{E}_{7}$ & $\mathcal{E}_{8}$ \\
\hline Composition & $\mathcal{H}_{0}$ & $\mathcal{H}_{1}$ & $\mathcal{H}_{0}{ }^{2}$ & $\mathcal{H}_{0}{ }^{3}, \mathcal{H}_{1}{ }^{2}$ & $\mathcal{H}_{0}{ }^{4}$ & $\mathcal{H}_{1}{ }^{3}$ & $\mathcal{H}_{0}{ }^{5}$ \\
Monodromy & $(S T)^{-1}$ & $(S)^{-1}$ & $(S T)^{-2}$ & $(S)^{2} \equiv-1$ & $(S T)^{2}$ & $S$ & $S T$ \\
Discrete Charge & $\mathbb{Z}_{6}$ & $\mathbb{Z}_{4}$ & $\mathbb{Z}_{3}$ & $\mathbb{Z}_{2}$ & $\mathbb{Z}_{3}$ & $\mathbb{Z}_{4}$ & $\mathbb{Z}_{6}$ \\
$\tau_{S}$ & $\rho$ & $i$ & $\rho$ & any & $\rho$ & $i$ & $\rho$ \\
\hline
\end{tabular}

Table 1: List of 7-planes with finite order monodromies in the z-plane, which do not carry net (ZZ-valued) $D$-brane charge. They support gauge fields on their world-volumina corresponding to the respective Kodaira singularity type $\left(\mathcal{H}_{n} \sim A_{n}\right)$. We also list their composition in terms of basic building blocks, as well as the associated constant type IIB string coupling, $\tau_{S}$. (S,T denote the standard generators of the $S$-duality group, $S L(2, \mathbb{Z})$.)

Because of the mutual locality, the anomalous couplings can be very simply determined. For this we recall that a $\mathcal{D}_{4}$-plane can be viewed as being composed out of four $D 7$ branes plus one orientifold plane, which all are mutually local. Since $\mathcal{L}_{O 7}^{(\mathrm{CS})}=-4 C \wedge$ $\left.\sqrt{\widehat{\mathcal{L}}(R)}\right|_{8 \text {-form }}$ [16, 17, 18], where $\widehat{\mathcal{L}}(R)$ is the Hirzebruch genus, we thus have:

$$
\begin{aligned}
\mathcal{L}_{\mathcal{D}_{4}}^{(\mathrm{CS})} & =\left.C \wedge\left[\operatorname{tr}\left(e^{-2 i F}\right) \wedge \sqrt{\widehat{\mathcal{A}}(R)}-4 \sqrt{\widehat{\mathcal{L}}(R)}\right]\right|_{8-\text { form }} \\
& =C^{(4)} \wedge\left(\frac{1}{2} R^{2}-2 \operatorname{tr} F^{2}\right)+C^{(0)} \wedge\left(\frac{2}{3} \operatorname{tr} F^{4}-\frac{1}{12} \operatorname{tr} F^{2} \operatorname{tr} R^{2}+\frac{1}{192}\left(\operatorname{tr} R^{2}\right)^{2}+\frac{1}{48} \operatorname{tr} R^{4}\right) \\
& =: 3 \mathcal{L}_{\mathcal{H}_{0}}^{(\mathrm{CS})}=: 2 \mathcal{L}_{\mathcal{H}_{1}}^{(\mathrm{CS})}
\end{aligned}
$$

where in the last line the gauge field traces follow implicitly from the decomposition $S O(8) \rightarrow U(1)$ or $S U(2)$, respectively. Indeed, summing over all world-volumina exactly reproduces the (eight dimensional remainder of the) Green-Schwarz term of the heterotic string, $\mathcal{L}^{(G S)}=C^{(6)} \wedge 2\left(R^{2}-\operatorname{tr} F_{S O(32)}^{2}\right)+C^{(2)} \wedge X_{8}\left(F_{S O(32)}, R\right)$.

\section{Geometric Interactions}

What we are interested in are the non-trivial interactions between the planes, which should ultimately reproduce the coupling functions $\tau_{\text {eff }}(T, U)$ that were schematically computed in (1). The primary perturbative contributions will arise from strings stretched between the various planes, which amounts to $C^{(p)}$ tensor field exchange between individual planes. The effective interaction will thus depend on the distances between the various 7-planes in the $z$-plane. More specifically, the closed string exchange that contributes to the maximal number of wedge products of field strengths is in the odd $R R$ sector, and is proportional to the Green's function $\Delta$ of a scalar field on the $z$-plane. Schematically:[]

$$
\begin{aligned}
\left\langle C_{m_{1} \ldots m_{p}}^{(p)}\left(z_{1}\right) C_{n_{1} \ldots n_{8-p}}^{(8-p)}\left(z_{2}\right)\right\rangle_{R R^{-}} & =\epsilon^{m_{1} \ldots m_{p} n_{1} \ldots n_{8-p}} \Delta\left(z_{1}, z_{2}\right), \\
\text { where } \Delta\left(z_{1}, z_{2}\right) & =\ln \left(z_{1}-z_{2}\right)+\text { finite } .
\end{aligned}
$$

The dependence of the plane locations $z_{i}$ on $T, U$ is complicated, but can be obtained via the $K 3$ mirror map, as explained in ref. [1, 10].

However, in order to obtain functionally exact results, we need to know the full Green's functions that probe the global structure of the $z$-plane, and not just their leading singular behavior. This is in general a complicated problem, but in our setup, where we consider

\footnotetext{
${ }^{2}$ For a more precise discussion, see eg. [17].
} 
only planes with finite order monodromies, there is a natural geometric answer [1], 10]. This is because the branch cuts in the z-plane are of finite order, and therefore the geometry is effectively the one of Riemann surfaces $\Sigma$ - see Fig.1 (indeed the geodesic lengths of strings stretching in the $z$-plane, given by $\int_{z_{1}}^{z_{2}} d z \prod_{i}\left(z-z_{i}\right)^{-1 / 12}$, turn out to be given by the periods of $\Sigma$ ).

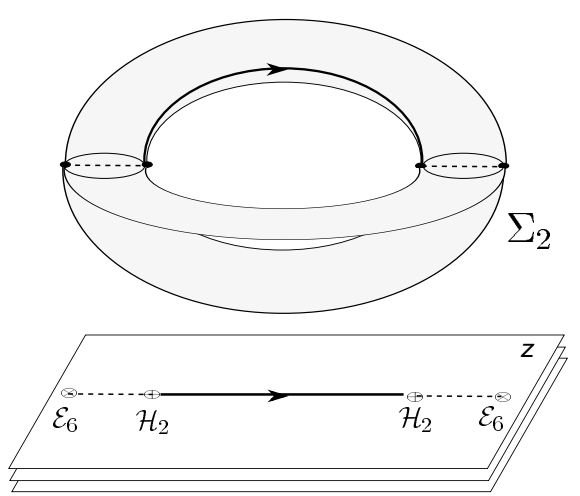

Figure 1: Lift of the $z$-plane to a covering Riemann surface. Shown is here the situation with two $\mathcal{E}_{6}$ and two $\mathcal{H}_{2}$ planes, which correspond to $\mathbb{Z}_{3}$ twist fields and anti-twist fields, respectively, located at the branch points of a genus two curve $\Sigma_{2}$. We also show a closed string trajectory that contributes to the coupling $\operatorname{tr} F_{S U(3)}{ }^{2} \operatorname{tr} F_{S U(3)^{\prime}}{ }^{2}$, and which corresponds to a $1 / 3-$ period on $\Sigma_{2}$.

Accordingly, canonical Green's functions are given by the ones of scalar fields on the covering surfaces $\Sigma$ : those are known to be given by the logarithm of the "prime form". However, it turns out [10] that these canonical Green's functions on $\Sigma$ to not capture the full story; they capture only the perturbative piece of the interactions. In fact, a Green's function is ambiguous up to the addition of finite pieces, and indeed there are in general further non-singular non-perturbative corrections to the naive Green's functions, arising from wrapping string world-sheets on $\Sigma$. It was shown in 10 how the non-perturbative Green's functions between pairs of branch points can be computed exactly by solving certain differential equations.

Due to lack of space, we will consider in the following mainly the interactions of model iii), which is Sen's model with $S O(8)^{4}$ gauge symmetry. Here four $\mathcal{D}_{4} 7$-branes create two $\mathbb{Z}_{2}$ branch cuts, so that the covering space is just a torus, $\Sigma=T^{2}$. The situation is especially simple also because the non-perturbative, $U$-dependent contributions factor out, so that the naive Green's functions on the torus give the full result (as far as the $T$ dependence is concerned ${ }^{3}$ ). More explicitly, the (harmonic parts of the) Green's functions between the four branch points are given by

$$
\Delta_{12}=\Delta_{34}=\ln \left[\frac{\theta_{2}(2 T)}{\theta_{1}^{\prime}(2 T)}\right], \quad \Delta_{13}=\Delta_{24}=\ln \left[\frac{\theta_{3}(2 T)}{\theta_{1}^{\prime}(2 T)}\right], \quad \Delta_{14}=\Delta_{23}=\ln \left[\frac{\theta_{4}(2 T)}{\theta_{1}^{\prime}(2 T)}\right] .
$$

Integrating out $C^{(4)}$ exchange between the Chern-Simons couplings in (3) therefore immediately yields the following terms in the effective action:

$$
\mathcal{L}^{\mathrm{eff}} \sim \sum_{i<j}^{4} \operatorname{Re}\left[\Delta_{i j}(T)\right] \operatorname{tr} F_{S O(8)_{i}}^{2} \wedge \operatorname{tr} F_{S O(8)_{j}}^{2} .
$$

These can be seen [1] to match exactly the heterotic one-loop couplings (with the appropriate Wilson lines switched on) ! Similarly, by summing up $C^{(4)}$ exchange between

\footnotetext{
${ }^{3}$ This is reflected by the fact that the $z$-plane geometry does not depend on the modulus $U$, which plays the role of $\tau_{S}$. Non-trivial $U$-dependence is induced by $D$-instantons, to the effect that $C^{(0)}$ in $(3)$ is renormalized into const $\times \ln |\eta(U)|^{4}$. This can be seen by comparing to the $U$-dependence as computed on the heterotic side [1].
} 
any given brane and all the other ones, the contribution to $\operatorname{tr} F_{S O(8)}^{2} \operatorname{tr} R^{2}$ is given by $\sum_{i=2}^{4} \Delta_{1 i}(T) \sim \ln [\eta(2 T)]$ - this again matches the heterotic result.

\section{Global Interactions from cyclic $D$-brane charges}

There are moduli-dependent corrections also to other eight-form terms in the effective action, eg., to $\left(\operatorname{tr} F^{2}\right)^{2}$ and $\operatorname{tr} R^{4}$ (again, we will focus here only on the perturbative, potentially singular contributions). In the usually considered situation, where one focuses on pairs of $D$ - or orientifold-branes [16, 17, 18], such terms arise from integrating out $C^{(0)}-C^{(8)}$ exchange between the two branes, each equipped with couplings like $\mathcal{L}^{(\mathrm{CS})}=$ $Q_{7} \cdot C^{(8)}+\ldots+C^{(0)} \wedge Y_{8}(F, R)$, where $Y_{8}$ is some 8-form. However, in the present context, the 7 -brane charge is cancelled locally on every plane so that $Q_{7} \equiv 0$. This means that naive $C^{(0)}-C^{(8)}$ exchange cannot contribute to these couplings, a fact that has been slightly mis-stated in ref. [四]. But how do these moduli-dependent corrections then arise?

The point is that despite our 7 -planes do not have net $\mathbb{Z}$-valued $D$-brane charge (no logarithmic monodromy), there is still a remnant left, which is reflected by the finite order $\mathbb{Z}_{N}$ monodromies. It is this "torsion" piece of the $D$-brane charge that is responsible for the requisite long-range interactions. This can be seen by analyzing the interactions in terms of string junctions [19] (with the appropriate quantum numbers so as to contribute to the terms in question). Similar to what is familiar from orientifold planes, what one finds are string trajectories that loop around other planes and do not couple to them via the local Chern-Simons terms in (3); see Fig.2.
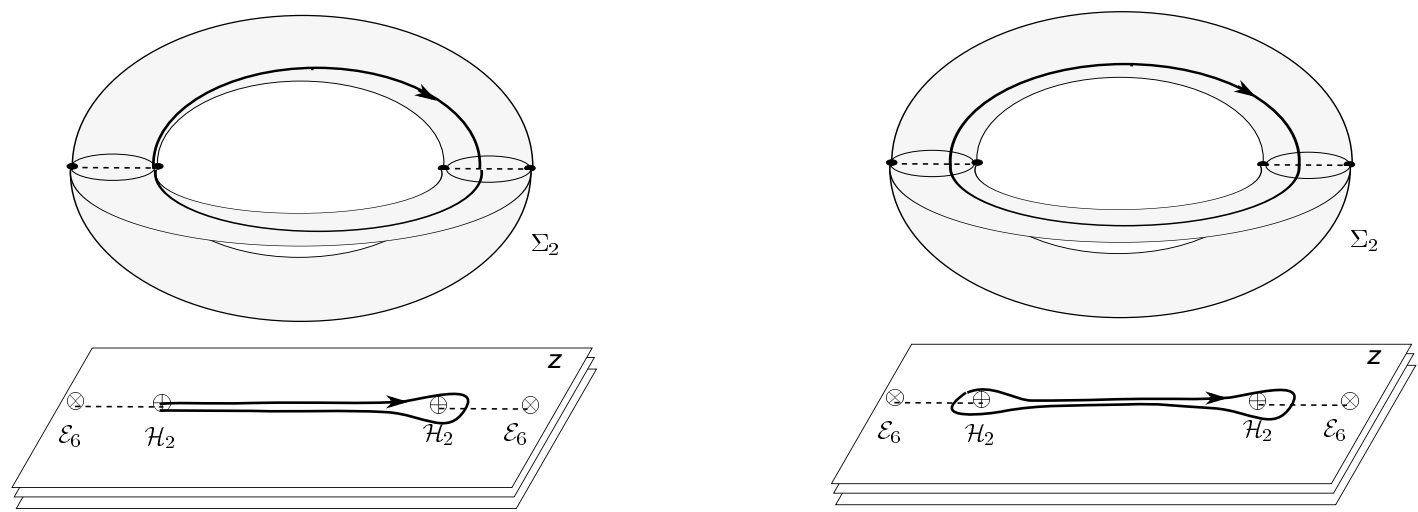

Figure 2: Interactions probing a $\mathbb{Z}_{3}$ torsion piece of $D$-brane charge. On the left, a string junction is shown that contributes to $\left(\operatorname{tr} F_{S U(3)}\right)^{2}$, while the one on the right contributes to $\operatorname{tr} R^{4}$. We also show how they lift to periods on the covering curve. The junctions give rise to logarithmic singularities when the planes collide (the actual BPS geodesics may look quite different, though, and might involve prongs.)

The question arises to what extent it is possible to represent such interactions in terms of local couplings on the plane world-volumina. One might imagine pulling the string loop through the plane location, thereby creating a prong that ends on the plane and thus might have a local coupling to it. Indeed for $\mathcal{D}_{4} 7$-planes, which are related to $\mathbb{Z}_{2}$ orientifold planes, the interaction can be treated in conformal field theory, where it can be represented by a cross cap vertex localized on the plane. On the other hand, for the $\mathbb{Z}_{N}$ generalizations listed in Table 1, there is no conformal field theory description because these 7-planes are associated with strong (and frozen) type IIB coupling, $\tau_{S}$. It thus seems more natural to view these interactions not in terms of localized couplings, but rather as 
analogous to those of "Alice strings" [20], which do have long-range interactions but no locally defined charge density.

At any rate, the heterotic string computation tells exactly what the answer must be, and it turns out that we obtain the correct result if we simply take the same Green's functions as before. That is, adding up all the relevant exchanges, we obtain $\sum_{i=2}^{4} \Delta_{1 i}(T) \sim$ $\ln [\eta(2 T)]\left(\operatorname{tr} F_{S O(8)_{1}}^{2}\right)^{2}$, and the same functional form as well for $\left(\operatorname{tr} R^{2}\right)^{2}$ and $\operatorname{tr} R^{4}$; up to normalization, this indeed reproduces the heterotic one-loop couplings. Note that the closed loops in the $z$-plane that contribute to the gravitational couplings lift to elliptic curves in the $K 3$, which is consistent with the expectation that gravitational couplings are not corrected at type IIB closed string tree level.
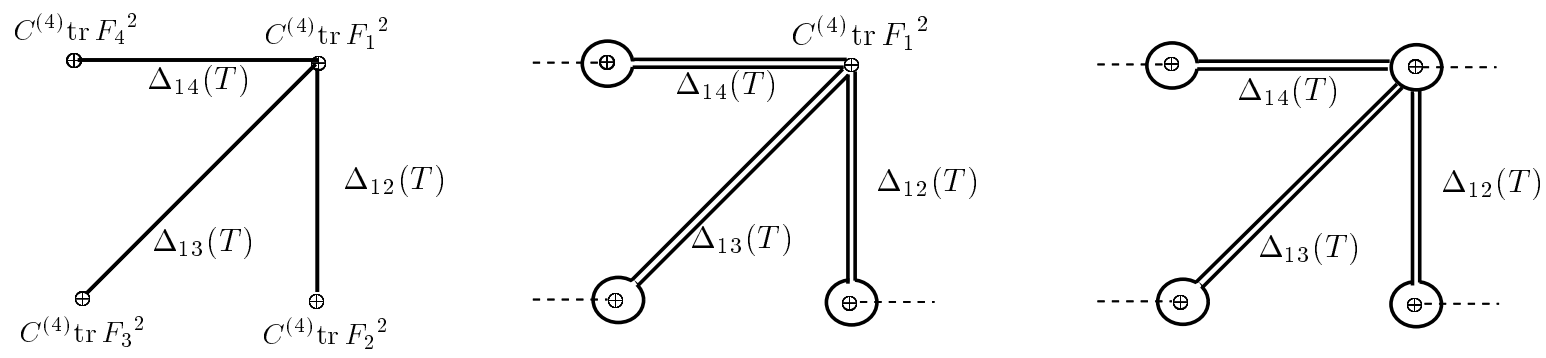

Figure 3: Comparison of some of the T-dependent interactions between $\mathcal{D}_{4}$ planes in the $S O(8)^{4}$ model. Shown on the left are the $C^{(4)}$ exchanges that couple locally and lead to the couplings $\operatorname{tr} F_{S O(8)_{1}}^{2} \operatorname{tr} F_{S O(8)_{j}}^{2}$. In the middle we see contributions to $\left(\operatorname{tr} F_{S O(8)_{1}}^{2}\right)^{2}$, and on the right some of the contributions to $\operatorname{tr} R^{4}$, the dashed lines denoting $\mathbb{Z}_{2}$ branch cuts.

We hope that the above considerations will be relevant in a broader context, perhaps in the $K$-theoretic approach [21] to generalized $D$-brane charges, where indeed $\mathbb{Z}_{N}$ charges play an important role. We may also present more detailed results elsewhere.

Acknowledgements We thank Nick Warner for collaboration on this subject and Peter Mayr for discussions, and moreover W.L. thanks the organizers of the Symposium for a pleasant workshop.

\section{References}

[1] W. Lerche and S. Stieberger, Prepotential, mirror map and F-theory on K3, hep-th/9804176, to appear in ATMP 2.5.

[2] C. Vafa, Evidence for F-theory, Nucl. Phys. B469 (1996) 403-418, hep-th/9602022.

[3] C. Bachas, C. Fabre, E. Kiritsis, N. Obers and P. Vanhove, Heterotic/type I duality and D-brane instantons, Nucl. Phys. B509 (1998) 33, hep-th/9707126.

[4] E. Kiritsis and N. Obers, Heterotic type I duality in $d<10$-dimensions, threshold corrections and D-instantons, JHEP 10 (1997) 004, hep-th/9709058.

[5] C. Bachas, Heterotic versus type-I, Nucl. Phys. Proc. Suppl. 68 (1998) 348, hep-th/9710102;

M. Bianchi, E. Gava, F. Morales and K. S. Narain, D strings in unconventional type I vacuum configurations, hep-th/9811013.

E. Gava, A. Hammou, J. F. Morales and K. S. Narain, On the perturbative corrections around D string instantons, hep-th/9902202. 
K. Foerger and S. Stieberger, Higher derivative couplings and heterotic type I duality in eight-dimensions, hep-th/9901020.

M. Gutperle, A Note on heterotic/type I' duality and D0 brane quantum mechanics, hep-th/9903010.

[6] W. Lerche, Elliptic index and superstring effective actions, Nucl. Phys. B308 (1988) 102.

[7] C. Bachas and E. Kiritsis, $F^{4}$ terms in $N=4$ string vacua, Nucl. Phys. Proc. Suppl. 55B (1997) 194, hep-th/9611205.

[8] A. Schellekens and N. Warner, Anomalies, characters and strings, Nucl. Phys. B287 (1987) 317;

E. Witten, Elliptic genera and quantum field theory, Comm. Math. Phys. 109 (1987) 525 ;

W. Lerche, B.E.W. Nilsson, A.N. Schellekens and N.P. Warner, Nucl. Phys. 299 (1988) 91.

[9] J. Harvey and G. Moore, Algebras, BPS States, and Strings, Nucl. Phys. B463 (1996) 315-368, hep-th/9510182.

[10] W. Lerche, S. Stieberger and N. Warner, Quartic gauge couplings from K3 geometry, hep-th/9811228.

[11] W. Lerche, S. Stieberger and N. Warner, Prepotentials from symmetric products, hep-th/9901162.

[12] M. Green, J. Harvey and G. Moore, I-brane inflow and anomalous couplings on dbranes, Class. Quant. Grav. 14 (1997) 47-52, hep-th/9605033.

[13] A. Sen, F-theory and Orientifolds, Nucl. Phys. B475(1 996) 562 hep-th/9605150.

[14] K. Dasgupta and S. Mukhi, F-theory at constant coupling, Phys. Lett. B385 (1996) 125-131, hep-th/9606044.

[15] K. Kodaira, Ann. Math. 77 (1963) 563; Ann. Math. 78 (1963) 1.

[16] K. Dasgupta, D. Jatkar and S. Mukhi, Gravitational couplings and $\mathbb{Z}_{2}$ orientifolds, Nucl. Phys. B523 (1998) 465, hep-th/9707224.

[17] J. Morales, C. Scrucca and M. Serone, Anomalous couplings for D-branes and Oplanes, hep-th/9812071.

[18] B. Craps and F. Roose, Anomalous D-brane and orientifold couplings from the boundary state, Phys. Lett. B445 (1998) 150, hep-th/9808074;

B. Stefanski, Gravitational couplings of D-branes and O-planes, hep-th/9812088.

[19] see eg.:

M. Gaberdiel, T. Hauer and B. Zwiebach, Open string-string junction transitions, Nucl. Phys. B525 (1998) 117, hep-th/9801205;

O. DeWolfe and B. Zwiebach, String junctions for arbitrary Lie algebra representations, Nucl. Phys. B541 (1999) 509, hep-th/9804210.

[20] A. S. Schwarz, Field theories with no local conservation of the electric charge, Nucl. Phys. B208 (1982) 141.

[21] E. Witten, D-branes and $K$ theory, hep-th/9810188. 